\title{
Determinants of child wasting in Bhutan. Insights from nationally representative data
}

\author{
Víctor M Aguayo ${ }^{1, *}$, Nina Badgaiyan ${ }^{1}$ and Laigden Dzed ${ }^{2}$ \\ 'UNICEF, Regional Office for South Asia, PO Box 5815, Lekhnath Marg, Kathmandu, Nepal: \\ ${ }^{2}$ Nutrition Programme, Ministry of Health, Royal Government of Bhutan, Thimphu, Bhutan
}

Submitted 25 January 2016: Final revision received 3 July 2016: Accepted 6 July 2016: First published online 30 August 2016

\begin{abstract}
Objective: To characterize the epidemiology of wasting and identify the main predictors of wasting, severe wasting and poor weight-for-height in children.

Design: We analysed a nationally representative sample of 2028 children (Multiple Indicator Survey, 2010).

Setting: Royal Kingdom of Bhutan.

Subjects: Children aged 0-23 months.

Results: Wasting prevalence was significantly higher among infants aged 0-11 months than among children aged 12-23 months $(12.0$ v. $6.7 \% ; P=0.004)$ and among boys than girls $(11.0 v .7 .5 \% ; P=0.04)$. Children from the Western region had $63 \%$ higher odds of being wasted than children from the Central/Eastern regions (adjusted OR $(\mathrm{AOR})=1.63 ; 95 \%$ CI 1.14, 2.34). Poor feeding practices were among the most significant predictors of wasting and severe wasting. Children who were given prelacteal feeds in the first days of life had 2.5 times higher odds of being severely wasted than those who were not $(\mathrm{AOR}=2.49 ; 95 \%$ CI $1.19,5 \cdot 19)$; inadequate complementary feeding in children aged 0-23 months was associated with $58 \%$ higher odds of being wasted (AOR $=1.58$; $95 \%$ CI 1.02, 2.47) and 2.3 times higher odds of being severely wasted ( $\mathrm{AOR}=2 \cdot 28$; $95 \% \mathrm{CI} 1.13,4.58$ ). The association of poor infant feeding practices with wasting and severe wasting was particularly significant in infants (0-11 months).

Conclusions: Programmes for the detection and treatment of severely wasted children need to prioritize very young children (0-11 months), particularly in the Western region. Programmes for the prevention of wasting need to prioritize the improvement of complementary foods and feeding practices in children aged 6-23 months.
\end{abstract}

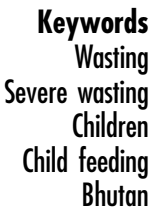

Weywords

wasting

Children

Bhutan
Globally, some 50 million children under 5 years of age (under-5s; $\sim 8 \%$ of children aged 0-59 months) suffer from wasting $^{(1)}$. Wasting (weight-for-height $<-2$ SD of the median weight-for-height in the WHO's Child Growth Standards) poses a serious threat to child survival and development ${ }^{(2)}$. Mortality rates in wasted children (weightfor-height $Z$-score (WHZ) <-2) and severely wasted children (WHZ <-3) are three to nine times higher than in children who are not wasted ${ }^{(3)}$.

Furthermore, wasted children who survive are at increased risk of stunted growth ${ }^{(4)}$. A recent analysis pooling data from eight longitudinal studies in Africa, Asia and Latin America indicated that children with highly variable weight-for-length $Z$-score, negative changes in weight-for-length $Z$-score and/or wasting in the first 17 months of life are at a higher risk of linear growth retardation and stunting at 18-24 months of age, which can result in adverse and often irreversible consequences, including poor cognition and learning performance, reduced lean body mass, short adult stature, lower productivity and reduced earnings ${ }^{(5-7)}$.

Despite recent economic growth in many countries, South Asia is the global epicentre of child wasting ${ }^{(8)}$. The latest data indicate that $16 \%$ of South Asia's under- 5 s are wasted. Levels of child wasting in South Asia are almost double those in sub-Saharan Africa (9\%) and four times higher than those in East Asia and the Pacific $(4 \%)^{(9)}$.

Landlocked at the eastern end of the Himalayas, Bhutan borders China to the north and India to the south, east and west. In 2010, Bhutan's Multiple Indicator Survey (BMIS) indicated that $6 \%$ of the country's children aged $0-59$ months were wasted and $2 \%$ were severely wasted ${ }^{(10)}$. The prevalence of wasting was more than twice higher among children aged 0-23 months than among children 
aged 24-59 months $(9 \cdot 2 v .3 \cdot 8 \%)$ while the prevalence of severe wasting was almost four times higher (3.8 v. 1.0\%). This is in line with global evidence indicating that most wasting happens during the $1000 \mathrm{~d}$ that span from conception until age 2 years ${ }^{(11)}$

The objective of the current analysis was threefold: (i) to characterize the epidemiology of wasting, severe wasting and poor weight-for-height in children aged 0-23 months in Bhutan; (ii) to identify the most significant predictors of wasting, severe wasting and poor weight-for-height in Bhutanese children aged 0-23 months; and (iii) to prioritize areas for action to prevent and treat child wasting in Bhutan.

\section{Methods}

We used publicly available data from the BMIS $2010^{(10)}$. BMIS - the customized version of the Multiple Indicator Cluster Survey (MICS) and the Demographic and Health Survey (DHS) - was a nationally representative household survey designed to provide estimates for indicators on the situation of children and women living in urban and rural areas in the three regions (Central, Eastern and Western) and twenty dzongkhags (districts) of the country.

A detailed description of the survey design, sampling methodology, survey tools and data collection can be found elsewhere ${ }^{(10)}$. In brief, the urban and rural areas within each dzongkhag were the main sampling strata. The sample was selected in two stages; within each stratum, a specified number of blocks in the urban areas and chiwogs (municipalities) in the rural areas were randomly selected as enumeration areas with probability proportional to size. Household listing was carried out within the selected enumeration areas and a systematic random sample of households was drawn in each enumeration area.

The survey used three questionnaires: (i) the household questionnaire, administered to collect information on all de jure (usual residents) household members, the household and the dwelling; (ii) the women questionnaire, administered in each household to all women aged 15-49 years; and (iii) the children questionnaire, administered to mothers/caregivers of children aged 0-59 months in the household. Once data collection processes were standardized, data collection took place from April to August 2010. Individual consent to participate in the survey was given by the child's caregiver. The survey included 15400 households, with a household response rate of $98.4 \%$ and a child response rate of $97.5 \%$. The survey received ethical clearance from the Research Ethics Board of Health and the National Statistical Bureau.

We selected data from the child data set for children aged 0-23 months and we adjusted for cluster sampling and sampling weights. Wasting and severe wasting were defined as the proportion of children whose WHZ was $<-2$ or $<-3$, respectively, using the WHO's Child Growth
Standards ${ }^{(2)}$. Analyses were performed using the Stata statistical software package release 12 (2011). In models that performed the regression of wasting or severe wasting as the outcome (dependent) variables $v$. exposure (independent) variables, we report adjusted odds ratios (AOR) and $95 \%$ confidence intervals from logistic regressions. In models that performed the regression of WHZ as the outcome variable $v$. exposure variables, we report regression coefficients and 95\% confidence intervals around point estimates from linear regressions. For all tests, $P$ values $<0.05$ were considered statistically significant. Our analysis did not require ethical approval as we used data that are available for public use and we ensured that data analysis was conducted anonymously.

\section{Results}

The survey included a representative sample of 2404 children aged 0-23 months. Our analytical sample included 2028 children (84.4\%) for whom information on weight and height - and therefore on WHZ, wasting and severe wasting - were available, after eliminating the records of children with missing or implausible anthropometric data $(\mathrm{WHZ}<-5$, WHZ $>+5)$.

\section{Sample characteristics}

The main characteristics of these children are summarized in Table 1: $8.3 \%$ were born with a low birth weight ( $<2500 \mathrm{~g}$ ); $33.5 \%$ were born to mothers who were married or entered a marital union before age 18 years; $37.0 \%$ lived in households that used unimproved sanitation facilities; $57 \cdot 8 \%$ were born to mothers whose delivery was not attended by a doctor, nurse, midwife or skilled personnel; and over $60 \%$ were born to mothers and/or fathers without formal education (62.5\% and $64.5 \%$, respectively).

The reported child feeding practices are presented in Table 2. Less than two-thirds (61.2\%) of children were breast-fed within $1 \mathrm{~h}$ of birth and less than half (39.9\%) of infants aged $0-5$ months were exclusively breast-fed; most children $(91.5 \%)$ continued to breast-feed at 1 year, while two-thirds $(66 \cdot 1 \%)$ continued to breast-feed at 2 years. Over two-thirds $(67 \cdot 1 \%)$ of children aged 0-23 months were breast-fed as recommended for their age. About three-quarters $(72.0 \%)$ of infants aged 6-8 months were fed complementary foods; a similar proportion (70.6\%) of children 6-23 months old were fed complementary foods a minimum number of times per day $(78.4 \%$ in infants 6-11 months old $v \cdot 67 \cdot 4 \%$ in children $12-23$ months old); and about one in ten children (11.1\%) aged 0-23 months was fed from a bottle with a nipple.

\section{Prevalence of wasting and severe wasting by child, mother and bousebold characteristics}

Tables 3-5 summarize the distribution of the three outcome variables - wasting, severe wasting and 
Table 1 Distribution of children 0-23 months old by socioeconomic characteristics. Bhutan, 2010

\begin{tabular}{|c|c|c|}
\hline & $\begin{array}{l}\text { Proportion } \\
\quad(\%)\end{array}$ & $\begin{array}{c}\text { Number } \\
(n)\end{array}$ \\
\hline \multicolumn{3}{|l|}{ Birth weight } \\
\hline Weighed at birth & $72 \cdot 0$ & 1380 \\
\hline Not weighed at birth & 28.0 & 536 \\
\hline Birth weight $\geq 2500 \mathrm{~g}$ & $91 \cdot 7$ & 1260 \\
\hline Birth weight $<2500 \mathrm{~g}$ & 8.3 & 113 \\
\hline \multicolumn{3}{|l|}{ Mother's age } \\
\hline $15-19$ years & $6 \cdot 4$ & 127 \\
\hline $20-24$ years & $29 \cdot 0$ & 576 \\
\hline $25-29$ years & 31.4 & 623 \\
\hline $29-39$ years & $28 \cdot 1$ & 558 \\
\hline$\geq 40$ years & $5 \cdot 1$ & 101 \\
\hline \multicolumn{3}{|l|}{ Parents' education } \\
\hline $\begin{array}{l}\text { Mother's education: No formal school } \\
\text { education }\end{array}$ & $62 \cdot 5$ & 1267 \\
\hline Mother's education: Primary education & 14.4 & 291 \\
\hline Mother's education: Secondary education & $23 \cdot 2$ & 470 \\
\hline $\begin{array}{l}\text { HH head's education: No formal school } \\
\text { education }\end{array}$ & 64.5 & 1302 \\
\hline HH head's education: Primary education & 14.5 & 292 \\
\hline $\begin{array}{l}\text { HH head's education: Secondary } \\
\text { education }\end{array}$ & $21 \cdot 0$ & 424 \\
\hline \multicolumn{3}{|l|}{ Number of $\mathrm{HH}$ members } \\
\hline$\leq 4$ & 28.7 & 580 \\
\hline$>4$ & $71 \cdot 3$ & 1438 \\
\hline \multicolumn{3}{|l|}{ Water, hygiene and sanitation in the $\mathrm{HH}$} \\
\hline $\begin{array}{l}\mathrm{HH} \text { uses improved sources of drinking- } \\
\text { water }\end{array}$ & $95 \cdot 9$ & 1936 \\
\hline $\begin{array}{l}\text { HH uses unimproved sources of drinking- } \\
\text { water }\end{array}$ & $4 \cdot 1$ & 82 \\
\hline $\mathrm{HH}$ uses an improved sanitation facility & 63.0 & 1272 \\
\hline $\mathrm{HH}$ uses an unimproved sanitation facility & $37 \cdot 0$ & 746 \\
\hline $\begin{array}{l}\mathrm{HH} \text { has a place for handwashing where } \\
\text { water and soap are available }\end{array}$ & $75 \cdot 6$ & 1528 \\
\hline $\begin{array}{l}\text { HH does not have a place for } \\
\text { handwashing with water and soap } \\
\text { available }\end{array}$ & $24 \cdot 4$ & 494 \\
\hline \multicolumn{3}{|l|}{ Mother's access to health services } \\
\hline $\begin{array}{l}\text { Mother (or her partner) uses a } \\
\text { contraceptive method }\end{array}$ & $64 \cdot 5$ & 1252 \\
\hline $\begin{array}{l}\text { Mother (or her partner) does not use a } \\
\text { contraceptive method }\end{array}$ & $35 \cdot 5$ & 687 \\
\hline $\begin{array}{l}\text { ANC during last pregnancy provided by a } \\
\text { doctor/nurse/midwife }\end{array}$ & $62 \cdot 7$ & 1173 \\
\hline $\begin{array}{l}\text { ANC during last pregnancy provided by } \\
\text { other than doctor/nurse/midwife }\end{array}$ & $37 \cdot 3$ & 697 \\
\hline ANC visits during last pregnancy $\leq 3$ & $22 \cdot 6$ & 420 \\
\hline ANC visits during last pregnancy $\geq 4$ & 77.4 & 1439 \\
\hline $\begin{array}{l}\text { Last delivery was assisted by a doctor/ } \\
\text { nurse/midwife/skilled staff }\end{array}$ & $42 \cdot 2$ & 810 \\
\hline $\begin{array}{l}\text { Last delivery was not assisted by a } \\
\text { doctor/nurse/midwife/skilled staff }\end{array}$ & $57 \cdot 8$ & 1111 \\
\hline $\begin{array}{l}\text { Last delivery took place in a health facility } \\
\text { (public or private) }\end{array}$ & $59 \cdot 8$ & 1149 \\
\hline $\begin{array}{l}\text { Last delivery took place at home/other } \\
\text { than a health facility }\end{array}$ & $40 \cdot 2$ & 771 \\
\hline
\end{tabular}

$\mathrm{HH}$, household; ANC antenatal care.

WHZ - by child (Table 3), maternal (Table 4) and household (Table 5) exposure variables.

Table 3 (child characteristics) indicates that the prevalence of wasting in children 0-23 months old was $9.3 \%$ while the prevalence of severe wasting was $3.5 \%$ : thus over one-third $(37 \cdot 6 \%)$ of the wasted children were severely wasted. The proportion of wasted children declined with age (from $14.7 \%$ in children aged
Table 2 Breast-feeding and complementary feeding practices in children 0-23 months old. Bhutan, 2010

\begin{tabular}{|c|c|c|}
\hline & $\begin{array}{l}\text { Proportion } \\
\quad(\%)\end{array}$ & $\begin{array}{c}\text { Number } \\
(n)\end{array}$ \\
\hline \multicolumn{3}{|l|}{ Breast-feeding practices } \\
\hline $\begin{array}{l}\text { Children } 0-23 \text { months old breast-fed } \\
\text { within } 1 \mathrm{~h} \text { of birth }\end{array}$ & $61 \cdot 2$ & 1165 \\
\hline $\begin{array}{l}\text { Children } 0-23 \text { months old breast-fed } \\
\text { within } 1 \mathrm{~d} \text { of birth }\end{array}$ & 93.4 & 1778 \\
\hline $\begin{array}{l}\text { Children } 0-23 \text { months old who received } \\
\text { prelacteal feeds }\end{array}$ & 8.6 & 176 \\
\hline $\begin{array}{l}\text { Children }<6 \text { months old who are } \\
\text { exclusively breast-fed }\end{array}$ & 39.9 & 177 \\
\hline $\begin{array}{l}\text { Children }<6 \text { months old who are } \\
\text { predominantly breast-fed }\end{array}$ & 58.5 & 260 \\
\hline $\begin{array}{l}\text { Children } 12-15 \text { months old who are } \\
\text { breast-fed }\end{array}$ & 91.5 & 324 \\
\hline $\begin{array}{l}\text { Children 20-23 months old who are } \\
\text { breast-fed }\end{array}$ & $66 \cdot 1$ & 222 \\
\hline $\begin{array}{l}\text { Children } 0-23 \text { months old who are } \\
\text { appropriately breast-fed }\end{array}$ & $67 \cdot 1$ & 1365 \\
\hline \multicolumn{3}{|l|}{ Complementary feeding practices } \\
\hline Children 6-8 months old who are fed CF & $72 \cdot 0$ & 217 \\
\hline $\begin{array}{l}\text { Children } 6-23 \text { months old who are breast- } \\
\text { fed and receive CF }\end{array}$ & $76 \cdot 1$ & 1206 \\
\hline $\begin{array}{l}\text { Children 6-23 months old who receive CF } \\
\text { a minimum number of times daily }\end{array}$ & $70 \cdot 6$ & 957 \\
\hline $\begin{array}{l}\text { Children } 6-11 \text { months old who receive CF } \\
\text { a minimum number of times daily }\end{array}$ & 78.4 & 309 \\
\hline $\begin{array}{l}\text { Children } 12-23 \text { months old who receive } \\
\text { CF a minimum number of times daily }\end{array}$ & $67 \cdot 4$ & 648 \\
\hline $\begin{array}{l}\text { Children 0-23 months old who are fed } \\
\text { from a bottle with a nipple }\end{array}$ & $11 \cdot 1$ & 224 \\
\hline
\end{tabular}

$\mathrm{CF}$, complementary foods.

0-5 months to $7 \cdot 2 \%$ in children aged 18-23 months; $P<0.005)$, as did the proportion of severely wasted children (from $7.4 \%$ in children aged $0-5$ months to $1.3 \%$ in children aged 18-23 months; $P=0 \cdot 003)$. The mean WHZ improved with age (from -0.17 in children aged 0-5 months to +0.04 in children aged 18-23 months; $P=0 \cdot 04)$.

The prevalence of wasting was significantly higher in boys than in girls $(11.0 v .7 .5 \% ; P=0.04)$ and in children 0 11 months old than in children 12-23 months old (12.0 $v$. $6.7 \%, P=0.004$; data not presented). The prevalence of severe wasting was significantly higher among children 0-11 months old than among children 12-23 months old ( 5.0 v. $2 \cdot 1 \%, P=0.009$; data not presented), in infants who were fed prelacteal feeds in the first days of life (8.1 v. $3 \cdot 0 \%$; $P=0.03)$ and in children aged $0-5$ months who were exclusively breast-fed (11.6 v. 3.8\%; $P=0.004)$. The mean WHZ was significantly poorer among children who were not weighed at birth $(P=0 \cdot 02)$, children born with a low weight $(<2500 \mathrm{~g} ; P<0.001)$, children $0-11$ months old $(P=0.04)$, and children $0-23$ months old who were breastfed as recommended for their age $(P=0.02)$.

Table 4 (maternal characteristics) indicates that the prevalence of severe wasting was significantly higher among children whose mothers received prenatal care by other than a doctor/nurse/midwife ( $4.4 v \cdot 2 \cdot 0 \% ; P=0 \cdot 01)$. The mean WHZ was significantly poorer among children 
Table 3 Prevalence of wasting, prevalence of severe wasting and mean weight-for-height Z-score (WHZ) in children 0-23 months old by child characteristics. Bhutan, 2010

\begin{tabular}{|c|c|c|c|c|c|}
\hline & $\begin{array}{l}\text { Proportion }(\%) \text { of } \\
\text { children wasted } \\
(W H Z<-2)\end{array}$ & $\begin{array}{c}\text { Proportion }(\%) \text { of } \\
\text { children severely } \\
\text { wasted }(\mathrm{WHZ}<-3)\end{array}$ & $\begin{array}{l}\text { Proportion (\%) of } \\
\text { wasted children who } \\
\text { are severely wasted }\end{array}$ & $\begin{array}{l}\text { Children's } \\
\text { mean } \\
\text { WHZ }\end{array}$ & $\begin{array}{c}\text { Number } \\
(n)\end{array}$ \\
\hline \multicolumn{6}{|l|}{ Sex } \\
\hline Male & $11 \cdot 0$ & $4 \cdot 1$ & $37 \cdot 3$ & -0.16 & 1047 \\
\hline Female & 7.5 & 2.9 & 38.7 & 0.01 & 981 \\
\hline$P$ value & 0.04 & 0.24 & & 0.09 & \\
\hline \multicolumn{6}{|l|}{ Age } \\
\hline $0-5$ months & 14.7 & 7.4 & $50 \cdot 3$ & -0.17 & 444 \\
\hline $6-11$ months & $9 \cdot 7$ & $2 \cdot 8$ & $28 \cdot 9$ & -0.07 & 522 \\
\hline $12-17$ months & $6 \cdot 3$ & $2 \cdot 8$ & 44.4 & -0.12 & 537 \\
\hline $18-23$ months & $7 \cdot 2$ & 1.3 & $18 \cdot 1$ & 0.04 & 525 \\
\hline$P$ value & 0.00 & 0.00 & & 0.04 & \\
\hline \multicolumn{6}{|l|}{ Birth weight } \\
\hline Weighed at birth & $9 \cdot 2$ & $3 \cdot 6$ & $39 \cdot 1$ & -0.01 & 1380 \\
\hline Not weighed at birth & $9 \cdot 7$ & 3.5 & $36 \cdot 1$ & -0.22 & 536 \\
\hline$P$ value & 0.72 & 0.60 & & 0.02 & \\
\hline Birth weight $\geq 2500 \mathrm{~g}$ & $9 \cdot 2$ & 3.3 & 35.9 & 0.03 & 1260 \\
\hline Birth weight $<2500 \mathrm{~g}$ & $15 \cdot 4$ & $6 \cdot 4$ & $41 \cdot 6$ & -0.45 & 113 \\
\hline$P$ value & 0.09 & 0.11 & & 0.00 & \\
\hline \multicolumn{6}{|l|}{ Breast-feeding practices } \\
\hline Breast-fed within $1 \mathrm{~h}$ of birth & $8 \cdot 8$ & $3 \cdot 1$ & $35 \cdot 4$ & 0.00 & 1165 \\
\hline Not breast-fed within $1 \mathrm{~h}$ of birth & $10 \cdot 7$ & $4 \cdot 2$ & $39 \cdot 3$ & -0.16 & 738 \\
\hline$P$ value & 0.67 & 0.49 & & 0.15 & \\
\hline Received prelacteal feeds & 10.9 & $8 \cdot 1$ & 74.3 & -0.30 & 1852 \\
\hline Did not receive prelacteal feeds & $9 \cdot 1$ & 3.0 & $33 \cdot 2$ & -0.06 & 176 \\
\hline$P$ value & 0.83 & 0.03 & & 0.50 & \\
\hline Is exclusively breast-fed (0-5 months) & $18 \cdot 6$ & 11.6 & 62.4 & -0.38 & 177 \\
\hline Is not exclusively breast-fed ( $0-5$ months) & $11 \cdot 2$ & $3 \cdot 8$ & 33.9 & 0.01 & 267 \\
\hline$P$ value & 0.14 & 0.004 & & 0.14 & \\
\hline Is predominantly breast-fed ( $0-5$ months) & $15 \cdot 9$ & 8.6 & $54 \cdot 1$ & -0.15 & 260 \\
\hline Is not predominantly breast-fed (0-5 months) & $12 \cdot 3$ & $5 \cdot 2$ & $42 \cdot 3$ & -0.22 & 184 \\
\hline$P$ value & 0.60 & $0 \cdot 16$ & & 0.57 & \\
\hline Is breast-fed (12-15 months) & $6 \cdot 4$ & 3.5 & 54.7 & -0.14 & 324 \\
\hline Is not breast-fed (12-15 months) & 2.9 & 2.9 & $100 \cdot 0$ & 0.67 & 30 \\
\hline$P$ value & 0.57 & 0.77 & & 0.02 & \\
\hline Is breast-fed (20-23 months) & $7 \cdot 4$ & 1.3 & $17 \cdot 6$ & -0.07 & 222 \\
\hline Is not breast-fed (20-23 months) & $10 \cdot 9$ & 0.0 & 0.0 & 0.18 & 114 \\
\hline$P$ value & 0.51 & 0.98 & & 0.38 & \\
\hline Is appropriately breast-fed (0-23 months) & $9 \cdot 2$ & 4.0 & 43.3 & -0.16 & 1345 \\
\hline Is not appropriately breast-fed (0-23 months) & $9 \cdot 4$ & $2 \cdot 4$ & $25 \cdot 2$ & 0.10 & 683 \\
\hline$P$ value & 0.33 & 0.33 & & 0.02 & \\
\hline \multicolumn{6}{|l|}{ Complementary feeding practices } \\
\hline Receives CF (6-8 months) & $12 \cdot 4$ & 4.7 & 37.9 & -0.16 & 214 \\
\hline Does not receive CF (6-8 months) & $9 \cdot 0$ & 0.0 & 0.1 & 0.36 & 83 \\
\hline$P$ value & 0.71 & 0.77 & & 0.27 & \\
\hline Is breast-fed and receives CF (6-23 months) & $7 \cdot 8$ & $2 \cdot 8$ & $35 \cdot 5$ & -0.11 & 1206 \\
\hline $\begin{array}{l}\text { Is not breast-fed and/or does not receive CF } \\
(6-23 \text { months) }\end{array}$ & 7.6 & 0.0 & 0.1 & 0.12 & 378 \\
\hline$P$ value & 0.88 & 0.82 & & 0.01 & \\
\hline $\begin{array}{l}\text { Receives CF a minimum number of times daily } \\
\text { (6-23 months) }\end{array}$ & $9 \cdot 3$ & $3 \cdot 1$ & 33.0 & -0.13 & 957 \\
\hline $\begin{array}{l}\text { Does not receive CF a minimum number of times } \\
\text { daily ( } 6-23 \text { months) }\end{array}$ & 3.7 & 0.01 & 0.2 & 0.01 & 399 \\
\hline$P$ value & 0.01 & $0 \cdot 18$ & & 0.18 & \\
\hline Is fed from a bottle with a nipple (0-23 months) & 8.7 & 3.0 & 34.9 & 0.03 & 224 \\
\hline $\begin{array}{l}\text { Is not fed from a bottle with a nipple } \\
\text { (0-23 months) }\end{array}$ & $9 \cdot 3$ & 3.5 & $37 \cdot 2$ & -0.09 & 1798 \\
\hline$P$ value & $0 \cdot 12$ & 0.27 & & 0.15 & \\
\hline Total & $9 \cdot 3$ & 3.5 & $37 \cdot 6$ & 0.20 & 2028 \\
\hline
\end{tabular}

CF, complementary foods.

born to mothers without any formal education $(P=0 \cdot 01)$ and children whose mothers were not married/in union at the time of the survey $(P=0.05$; data not presented).

Table 5 (household characteristics) indicates that the prevalence of wasting and severe wasting were significantly higher (i.e. double) in the Western region than in the Central and Eastern regions (12.8 v. 6.1 and $6.4 \%, P=0.002$ and 4.9 v. 2.9 and $1.6 \%, P=0.07$ for wasting and severe wasting in the Western, Central and Eastern region, respectively). The prevalence of wasting 
Table 4 Prevalence of wasting, prevalence of severe wasting and mean weight-for-height $Z$-score (WHZ) in children 0-23 months old by maternal characteristics. Bhutan, 2010

\begin{tabular}{|c|c|c|c|c|c|}
\hline & $\begin{array}{l}\text { Proportion }(\%) \text { of } \\
\text { children wasted } \\
(\mathrm{WHZ}<-2)\end{array}$ & $\begin{array}{c}\text { Proportion }(\%) \text { of } \\
\text { children severely } \\
\text { wasted }(\mathrm{WHZ}<-3)\end{array}$ & $\begin{array}{l}\text { Proportion (\%) of } \\
\text { wasted children who } \\
\text { are severely wasted }\end{array}$ & $\begin{array}{l}\text { Children's } \\
\text { mean WHZ }\end{array}$ & $\underset{(n)}{\text { Number }}$ \\
\hline \multicolumn{6}{|l|}{ Mother's age } \\
\hline $15-19$ years & $11 \cdot 3$ & 1.7 & $15 \cdot 0$ & $-0 \cdot 10$ & 127 \\
\hline $20-24$ years & $8 \cdot 0$ & 4.3 & $54 \cdot 0$ & 0.03 & 576 \\
\hline $25-29$ years & 9.6 & 3.7 & 38.4 & -0.09 & 623 \\
\hline $29-39$ years & $10 \cdot 4$ & 3.5 & 33.2 & $-0 \cdot 19$ & 558 \\
\hline$\geq 40$ years & 8.9 & 0.0 & 0.0 & 0.13 & 101 \\
\hline$P$ value & 0.73 & 0.27 & & 0.14 & \\
\hline \multicolumn{6}{|l|}{ Mother's education } \\
\hline None & $9 \cdot 4$ & $3 \cdot 8$ & $40 \cdot 4$ & $-0 \cdot 15$ & 1267 \\
\hline Primary & 8.0 & $2 \cdot 3$ & 28.8 & -0.01 & 291 \\
\hline Secondary & $9 \cdot 7$ & $3 \cdot 1$ & $31 \cdot 6$ & 0.06 & 470 \\
\hline$P$ value & 0.77 & 0.57 & & 0.01 & \\
\hline \multicolumn{6}{|l|}{ Mother (or her partner)'s use of contraception } \\
\hline Mother (or her partner) uses a contraceptive method & $9 \cdot 1$ & $3 \cdot 0$ & 33.0 & -0.07 & 1252 \\
\hline $\begin{array}{l}\text { Mother (or her partner) does not use a contraceptive } \\
\text { method }\end{array}$ & $10 \cdot 2$ & $4 \cdot 7$ & $46 \cdot 1$ & -0.08 & 687 \\
\hline \multirow{2}{*}{\multicolumn{6}{|c|}{ Mother's use of ANC }} \\
\hline & & & & & \\
\hline ANC was provided by a doctor/nurse/midwife & $7 \cdot 2$ & $2 \cdot 0$ & $27 \cdot 6$ & $-0 \cdot 10$ & 1173 \\
\hline $\begin{array}{l}\text { ANC was provided by other than a doctor/nurse/ } \\
\text { midwife }\end{array}$ & $10 \cdot 3$ & $4 \cdot 4$ & $42 \cdot 2$ & 0.01 & 697 \\
\hline$P$ value & 0.07 & 0.01 & & 0.33 & \\
\hline Number of ANC visits during the last pregnancy $\leq 3$ & $9 \cdot 2$ & 4.6 & $50 \cdot 0$ & -0.15 & 420 \\
\hline Number of ANC visits during the last pregnancy $\geq 4$ & $9 \cdot 3$ & $3 \cdot 3$ & 35.5 & -0.04 & 1439 \\
\hline$P$ value & 0.38 & 0.66 & & & 0.70 \\
\hline \multicolumn{6}{|l|}{ Mother's assistance at/place of delivery } \\
\hline $\begin{array}{l}\text { Mother's last delivery was assisted by a doctor/ } \\
\text { nurse/midwife }\end{array}$ & $8 \cdot 4$ & $3 \cdot 3$ & $39 \cdot 3$ & 0.00 & 810 \\
\hline $\begin{array}{l}\text { Mother's last delivery was not assisted by a doctor/ } \\
\text { nurse/midwife }\end{array}$ & $10 \cdot 3$ & $3 \cdot 7$ & 35.9 & -0.11 & 1111 \\
\hline \multirow{2}{*}{\multicolumn{6}{|c|}{ Mother's place of delivery }} \\
\hline & & & & & \\
\hline $\begin{array}{l}\text { Mother delivered last child in a health facility (public } \\
\text { or private) }\end{array}$ & $9 \cdot 4$ & 3.5 & $37 \cdot 2$ & -0.02 & 1149 \\
\hline $\begin{array}{l}\text { Mother delivered last child at home/not in a facility } \\
\text { (public or private) }\end{array}$ & $9 \cdot 7$ & $3 \cdot 6$ & $37 \cdot 1$ & -0.14 & 771 \\
\hline$P$ value & 0.38 & 0.31 & & 0.09 & \\
\hline \multicolumn{6}{|l|}{ Under-age marriage } \\
\hline $\begin{array}{l}\text { Mother was married or entered a marital union before } \\
\text { age } 18 \text { years }\end{array}$ & $8 \cdot 8$ & $3 \cdot 3$ & 37.5 & -0.07 & 659 \\
\hline $\begin{array}{l}\text { Mother was married or entered marital union at age } \\
\geq 18 \text { years }\end{array}$ & $9 \cdot 8$ & $3 \cdot 7$ & $37 \cdot 8$ & -0.08 & 1306 \\
\hline$P$ value & 0.51 & 0.60 & & 0.31 & \\
\hline \multicolumn{6}{|l|}{ Domestic violence } \\
\hline $\begin{array}{l}\text { Mother believes husband is justified in beating his } \\
\text { wife/partner }\end{array}$ & $8 \cdot 6$ & $2 \cdot 9$ & 33.7 & -0.07 & 1415 \\
\hline $\begin{array}{l}\text { Mother believes husband is not justified in beating } \\
\text { his wife/partner }\end{array}$ & $11 \cdot 1$ & $5 \cdot 2$ & $46 \cdot 4$ & -0.08 & 525 \\
\hline$P$ value & 0.21 & 0.27 & & 0.90 & \\
\hline Total & $9 \cdot 3$ & 3.5 & 37.6 & 0.20 & 2028 \\
\hline
\end{tabular}

ANC antenatal care.

tended to be significantly higher in urban than in rural areas $(11 \cdot 1 \quad v .8 .5 \% ; P=0.05)$. The mean WHZ was significantly poorer in children from the Western region $(P=0.01)$ and children from the two lowest wealth quintiles $(P<0 \cdot 01)$.

\section{Multivariate regression analysis: main predictors of child wasting in Bbutan}

Multivariate logistic regression - after adjusting for age, sex, residence and selected caregiver and household-level variables - indicated that three variables were independently associated with wasting in children 0-23 months old: age, region and complementary feeding (Table 6). Children aged 0-11 months had a $46 \%$ higher odds of being wasted than children aged 12-23 months (AOR= $1 \cdot 46 ; 95 \%$ CI 1.00, 2.13). Children aged 0-23 months from the Western region had $63 \%$ higher odds of being wasted than those from the Central and Eastern regions (AOR $=$ $1 \cdot 63$; $95 \%$ CI 1.14, 2.34), while children aged 0-11 months from the Western region had a 2-2-fold higher odds of 
Table 5 Prevalence of wasting, prevalence of severe wasting and mean weight-for-height $Z$-score (WHZ) in children 0-23 months old by household characteristics. Bhutan, 2010

\begin{tabular}{|c|c|c|c|c|c|}
\hline & $\begin{array}{c}\text { Proportion (\%) of } \\
\text { children wasted } \\
(\mathrm{WHZ}<-2)\end{array}$ & $\begin{array}{c}\text { Proportion (\%) of children } \\
\text { severely wasted } \\
(\mathrm{WHZ}<-3)\end{array}$ & $\begin{array}{c}\text { Proportion (\%) of } \\
\text { wasted children who } \\
\text { are severely wasted }\end{array}$ & $\begin{array}{l}\text { Children's } \\
\text { mean } \\
\text { WHZ }\end{array}$ & $\begin{array}{c}\text { Number } \\
(n)\end{array}$ \\
\hline \multicolumn{6}{|l|}{ Residence } \\
\hline Urban & $11 \cdot 1$ & 4.7 & $42 \cdot 3$ & 0.01 & 456 \\
\hline Rural & 8.5 & 2.9 & $34 \cdot 1$ & -0.12 & 1572 \\
\hline$P$ value & 0.05 & 0.3 & & 0.31 & \\
\hline \multicolumn{6}{|l|}{ Region } \\
\hline Western & $12 \cdot 8$ & 4.9 & $38 \cdot 3$ & -0.24 & 661 \\
\hline Central & $6 \cdot 1$ & 2.9 & 47.5 & -0.02 & 826 \\
\hline Eastern & $6 \cdot 4$ & 1.6 & $25 \cdot 0$ & 0.13 & 541 \\
\hline$P$ value & 0.002 & 0.07 & & 0.01 & \\
\hline \multicolumn{6}{|l|}{ Wealth index } \\
\hline Poorest & $7 \cdot 1$ & 1.9 & $26 \cdot 8$ & -0.14 & 404 \\
\hline Second & 11.8 & 3.0 & $25 \cdot 4$ & -0.21 & 435 \\
\hline Middle & 7.9 & 4.2 & 53.2 & 0.06 & 429 \\
\hline Fourth & 8.9 & $2 \cdot 8$ & 31.5 & -0.04 & 400 \\
\hline Richest & 11.3 & 5.7 & $50 \cdot 4$ & -0.05 & 317 \\
\hline$P$ value & 0.63 & 0.12 & & 0.00 & \\
\hline \multicolumn{6}{|l|}{$\mathrm{HH}$ head's education } \\
\hline None & 8.2 & $2 \cdot 7$ & $32 \cdot 9$ & -0.04 & 1302 \\
\hline Primary & 8.7 & $4 \cdot 3$ & 49.4 & -0.22 & 292 \\
\hline Secondary & $12 \cdot 4$ & 4.8 & 38.7 & -0.09 & 424 \\
\hline$P$ value & 0.08 & 0.05 & & 0.26 & \\
\hline \multicolumn{6}{|l|}{ Number of $\mathrm{HH}$ members } \\
\hline$\leq 4$ & 9.0 & $3 \cdot 3$ & $36 \cdot 7$ & -0.01 & 580 \\
\hline$>4$ & 9.4 & 3.5 & $37 \cdot 2$ & -0.11 & 1438 \\
\hline$P$ value & 0.85 & 0.88 & & 0.21 & \\
\hline \multicolumn{6}{|l|}{ Water, hygiene and sanitation in $\mathrm{HH}$} \\
\hline $\begin{array}{l}\text { HH uses improved sources of } \\
\text { drinking-water }\end{array}$ & $9 \cdot 0$ & $3 \cdot 2$ & $35 \cdot 6$ & -0.06 & 1936 \\
\hline $\begin{array}{l}\text { HH uses unimproved sources of } \\
\text { drinking-water }\end{array}$ & $15 \cdot 9$ & $10 \cdot 1$ & $63 \cdot 5$ & -0.44 & 82 \\
\hline$P$ value & 0.44 & $0 \cdot 10$ & & 0.43 & \\
\hline $\begin{array}{l}\text { HH uses improved sanitation } \\
\text { facilities }\end{array}$ & 9.6 & 3.9 & $40 \cdot 6$ & -0.08 & 1272 \\
\hline $\begin{array}{l}\text { HH uses unimproved sanitation } \\
\text { facilities }\end{array}$ & 8.7 & $2 \cdot 5$ & 28.7 & -0.07 & 746 \\
\hline$P$ value & 0.54 & 0.14 & & 0.15 & \\
\hline $\begin{array}{l}\mathrm{HH} \text { has a place for handwashing } \\
\text { where water and soap are available }\end{array}$ & 9.8 & $3 \cdot 3$ & 33.7 & -0.08 & 1528 \\
\hline $\begin{array}{l}\text { HH does not have a place for } \\
\text { handwashing with water and soap } \\
\text { available }\end{array}$ & $6 \cdot 1$ & 3.9 & 63.9 & -0.04 & 494 \\
\hline$P$ value & 0.36 & 0.35 & & 0.60 & 2022 \\
\hline Total & $9 \cdot 3$ & 3.5 & 37.6 & 0.20 & 2028 \\
\hline
\end{tabular}

$\mathrm{HH}$, household.

being wasted than those from the Central/Eastern regions $(\mathrm{AOR}=2 \cdot 24 ; 95 \% \mathrm{CI} 1 \cdot 39,3 \cdot 60)$. Finally, the odds of being wasted were $58 \%$ higher in children 0-23 months old who were not fed complementary foods as appropriate for their age $(\mathrm{AOR}=1.58 ; 95 \% \mathrm{CI} 1.02,2.47)$; this association was even stronger in children aged $0-11$ months as the odds of being wasted were about twofold higher in children aged 0-11 months old who were not fed complementary foods as appropriate for their age $(\mathrm{AOR}=1 \cdot 96 ; 95 \%$ CI $1 \cdot 21$, 3.16; Table 6).

Four variables were independently associated with severe wasting in children $0-23$ months old: antenatal care, prelacteal feeding, breast-feeding and complementary feeding. The odds of being severely wasted were $2 \cdot 2$-fold higher in children aged $0-23$ months whose mothers did not receive antenatal care by a doctor, nurse or midwife $(\mathrm{AOR}=2.21 ; 95 \% \mathrm{CI} 1.13,4.33)$; similarly, the odds of being severely wasted were $2 \cdot 5$-fold higher in children who were fed prelacteal feeds $(\mathrm{AOR}=2.49 ; 95 \%$ CI $1.19,5.19)$ and $2 \cdot 3$-fold higher among children who were not fed complementary foods as appropriate for their age $(\mathrm{AOR}=2 \cdot 28 ; 95 \% \mathrm{CI} 1 \cdot 13,4.58)$. The association of infant feeding variables with severe wasting was particularly strong in children $0-11$ months old; the odds of being severely wasted were fourfold higher in children 0-11 months old who were given prelacteal feeds $(\mathrm{AOR}=4.33 ; 95 \% \mathrm{CI} 1.76,10.66)$ and threefold higher among those who were not fed complementary foods as appropriate for their age (AOR $=2.94 ; 95 \%$ CI 1.42, 6.08). Breast-feeding was associated with a higher odds of 
Table 6 Adjusted odds ratios (AOR) of wasting and severe wasting by age group in relation to child, maternal and household characteristics. Bhutan, 2010

\begin{tabular}{|c|c|c|c|c|c|c|c|c|c|c|c|c|}
\hline & \multicolumn{6}{|c|}{ Wasting } & \multicolumn{6}{|c|}{ Severe wasting } \\
\hline & \multicolumn{2}{|c|}{$0-11$ months } & \multicolumn{2}{|c|}{ 12-23 months } & \multicolumn{2}{|c|}{$0-23$ months } & \multicolumn{2}{|c|}{$0-11$ months } & \multicolumn{2}{|c|}{$12-23$ months } & \multicolumn{2}{|c|}{$0-23$ months } \\
\hline & AOR & $95 \% \mathrm{Cl}$ & AOR & $95 \% \mathrm{Cl}$ & AOR & $95 \% \mathrm{Cl}$ & AOR & $95 \% \mathrm{Cl}$ & AOR & $95 \% \mathrm{Cl}$ & AOR & $95 \% \mathrm{Cl}$ \\
\hline \multicolumn{13}{|l|}{ Age } \\
\hline $\begin{array}{l}12-23 \text { months } \\
0-11 \text { months }\end{array}$ & $\begin{array}{l}1.0 \\
\mathrm{n} / \mathrm{a}\end{array}$ & $\begin{array}{l}\text { Ref. } \\
\text { n/a }\end{array}$ & $\begin{array}{l}1.0 \\
\mathrm{n} / \mathrm{a}\end{array}$ & $\begin{array}{l}\text { Ref. } \\
\text { n/a }\end{array}$ & $\begin{array}{l}1.0 \\
1.46\end{array}$ & $\begin{array}{c}\text { Ref. } \\
1 \cdot 00,2 \cdot 13\end{array}$ & $\begin{array}{l}1.0 \\
\mathrm{n} / \mathrm{a}\end{array}$ & $\begin{array}{l}\text { Ref. } \\
\text { n/a }\end{array}$ & $\begin{array}{l}1.0 \\
\mathrm{n} / \mathrm{a}\end{array}$ & $\begin{array}{l}\text { Ref. } \\
\text { n/a }\end{array}$ & $\begin{array}{l}1.0 \\
1.59\end{array}$ & $\begin{array}{c}\text { Ref. } \\
0.87,2.91\end{array}$ \\
\hline \multicolumn{13}{|l|}{ Sex } \\
\hline $\begin{array}{l}\text { Female } \\
\text { Male }\end{array}$ & $\begin{array}{l}1 \cdot 0 \\
1.23\end{array}$ & $\begin{array}{c}\text { Ref. } \\
0.78,1.96\end{array}$ & $\begin{array}{l}1.0 \\
1.52\end{array}$ & $\begin{array}{c}\text { Ref. } \\
0.87,2.67\end{array}$ & $\begin{array}{l}1.0 \\
1.35\end{array}$ & $\begin{array}{c}\text { Ref. } \\
0.95,1.92\end{array}$ & $\begin{array}{l}1.0 \\
1.34\end{array}$ & $\begin{array}{c}\text { Ref. } \\
0.66,2.68\end{array}$ & $\begin{array}{l}1.0 \\
1.03\end{array}$ & $\begin{array}{c}\text { Ref. } \\
0.43,2.49\end{array}$ & $\begin{array}{l}1.0 \\
1.20\end{array}$ & $\begin{array}{c}\text { Ref. } \\
0.70,2.05\end{array}$ \\
\hline \multicolumn{13}{|c|}{ 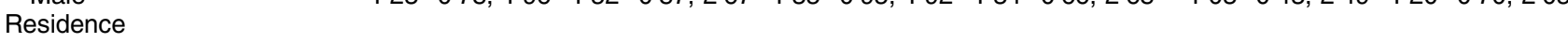 } \\
\hline $\begin{array}{l}\text { Urban } \\
\text { Rural }\end{array}$ & $\begin{array}{l}1 \cdot 0 \\
0.84\end{array}$ & $\begin{array}{c}\text { Ref. } \\
0.47,1.50\end{array}$ & $\begin{array}{l}1.0 \\
0.76\end{array}$ & $\begin{array}{c}\text { Ref. } \\
0.39,1.47\end{array}$ & $\begin{array}{l}1.0 \\
0.79\end{array}$ & $\begin{array}{c}\text { Ref. } \\
0.52,1.20\end{array}$ & $\begin{array}{l}1 \cdot 0 \\
1.32\end{array}$ & $\begin{array}{c}\text { Ref. } \\
0.56,3 \cdot 14\end{array}$ & $\begin{array}{l}1.0 \\
1.46\end{array}$ & $\begin{array}{c}\text { Ref. } \\
0.47,4.59\end{array}$ & $\begin{array}{l}1.0 \\
1.30\end{array}$ & $\begin{array}{c}\text { Ref. } \\
0.65,2.59\end{array}$ \\
\hline \multicolumn{13}{|c|}{ 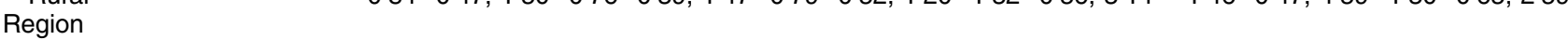 } \\
\hline $\begin{array}{l}\text { Central/Eastern } \\
\text { Western } \\
\text { HH head's education }\end{array}$ & $\begin{array}{l}1 \cdot 0 \\
2 \cdot 24\end{array}$ & $\begin{array}{c}\text { Ref. } \\
1.39,3.60\end{array}$ & $\begin{array}{l}1.0 \\
1.05\end{array}$ & $\begin{array}{c}\text { Ref. } \\
0.57,1.92\end{array}$ & $\begin{array}{l}1.0 \\
1.63\end{array}$ & $\begin{array}{c}\text { Ref. } \\
1 \cdot 14,2 \cdot 34\end{array}$ & $\begin{array}{l}1.0 \\
1.51\end{array}$ & $\begin{array}{c}\text { Ref. } \\
0.74,3.08\end{array}$ & $\begin{array}{l}1.0 \\
0.86\end{array}$ & $\begin{array}{c}\text { Ref. } \\
0.34,2 \cdot 17\end{array}$ & $\begin{array}{l}1.0 \\
1.25\end{array}$ & $\begin{array}{c}\text { Ref. } \\
0.72,2 \cdot 18\end{array}$ \\
\hline $\begin{array}{l}\text { Secondary } \\
\text { None }\end{array}$ & $\begin{array}{l}1.0 \\
1.03\end{array}$ & $\begin{array}{c}\text { Ref. } \\
0.58,1.83\end{array}$ & $\begin{array}{l}1.0 \\
0.75\end{array}$ & $\begin{array}{c}\text { Ref. } \\
0.38,1.49\end{array}$ & $\begin{array}{l}1.0 \\
0.91\end{array}$ & $\begin{array}{c}\text { Ref. } \\
0.59,1.40\end{array}$ & $\begin{array}{l}1.0 \\
0.99\end{array}$ & $\begin{array}{c}\text { Ref. } \\
0.43,2.29\end{array}$ & $\begin{array}{l}1.0 \\
0.43\end{array}$ & $\begin{array}{c}\text { Ref. } \\
0.15,1.20\end{array}$ & $\begin{array}{l}1.0 \\
0.72\end{array}$ & $\begin{array}{c}\text { Ref. } \\
0.36,1.43\end{array}$ \\
\hline $\begin{array}{l}\text { Who provides ANC } \\
\text { ANC provided by a doctor/ } \\
\text { nurse/midwife }\end{array}$ & $1 \cdot 0$ & Ref. & $1 \cdot 0$ & Ref. & 1.0 & Ref. & $1 \cdot 0$ & Ref. & 1.0 & Ref. & $1 \cdot 0$ & Ref. \\
\hline $\begin{array}{l}\text { ANC provided by other } \\
\text { than doctor/nurse/midwife }\end{array}$ & 1.22 & $0.72,2.08$ & 1.08 & $0.58,2.01$ & $1 \cdot 18$ & $0.66,2 \cdot 11$ & $2 \cdot 31$ & $1 \cdot 01,5 \cdot 36$ & $2 \cdot 05$ & $0.63,6.66$ & $2 \cdot 21$ & $1 \cdot 13,4.33$ \\
\hline \multicolumn{13}{|l|}{ Prelacteal feeding (0-23 months) } \\
\hline $\begin{array}{l}\text { Did not receive prelacteal } \\
\text { feeds }\end{array}$ & 1.0 & Ref. & 1.0 & Ref. & 1.0 & Ref. & 1.0 & Ref. & 1.0 & Ref. & 1.0 & Ref. \\
\hline $\begin{array}{l}\text { Received prelacteal feeds } \\
\text { Age-appropriate breast-feeding }\end{array}$ & 1.49 & $0.68,3.25$ & 0.86 & $0 \cdot 34,2 \cdot 17$ & $1 \cdot 22$ & $0.68,2 \cdot 18$ & 4.33 & $1 \cdot 76,10 \cdot 66$ & 0.93 & $0 \cdot 21,4 \cdot 10$ & $2 \cdot 49$ & $1.19,5 \cdot 19$ \\
\hline $\begin{array}{l}\text { Children are not breast-fed as } \\
\text { appropriate for their age }\end{array}$ & 1.0 & Ref. & 1.0 & Ref. & 1.0 & Ref. & 1.0 & Ref. & 1.0 & Ref. & 1.0 & Ref. \\
\hline $\begin{array}{l}\text { Children are breast-fed } \\
\text { as appropriate for their age }\end{array}$ & 1.07 & $0.65,1.78$ & 0.64 & $0.32,1 \cdot 26$ & 0.98 & $0.64,1.19$ & 2.51 & $1 \cdot 13,5 \cdot 51$ & 0.62 & $0.21,1.79$ & 1.79 & $0.87,3.69$ \\
\hline \multicolumn{13}{|l|}{$\begin{array}{l}\text { Age-appropriate } \\
\text { complementary feeding }\end{array}$} \\
\hline $\begin{array}{l}\text { Child is fed CF as } \\
\text { appropriate for age }\end{array}$ & 1.0 & Ref. & 1.0 & Ref. & 1.0 & Ref. & 1.0 & Ref. & 1.0 & Ref. & 1.0 & Ref. \\
\hline $\begin{array}{l}\text { Child is not fed CF as } \\
\text { appropriate for age }\end{array}$ & 1.96 & $1 \cdot 21,3 \cdot 16$ & 0.59 & $0 \cdot 18,1 \cdot 88$ & 1.58 & $1.02,2.47$ & 2.94 & $1.42,6.08$ & 0.42 & $0.05,3.70$ & $2 \cdot 28$ & $1.13,4.58$ \\
\hline
\end{tabular}

$\mathrm{HH}$, household; ANC, antenatal care; CF, complementary foods; ref., reference category.

severe wasting in infants $0-11$ months old $(\mathrm{AOR}=2 \cdot 51$; $95 \%$ CI $1 \cdot 13,5 \cdot 51$; Table 6).

The models performing the regression of $\mathrm{WHZ}$ on the exposure variables indicated that the likelihood of poor WHZ was significantly higher among children who were not weighed at birth $(P<0 \cdot 05)$, children of mothers without formal education $(P<0 \cdot 01)$ and children from the Western region $(P<0 \cdot 01$; Table 7$)$.

\section{Discussion}

Between 1988 and 2010, the prevalence of wasting in Bhutanese children aged 0-59 months hovered around $6 \%$ without much improvement, with levels of wasting and severe wasting two to four times higher among children aged 0-23 months than among children aged 24-59 months ${ }^{(10,12)}$. We used data from BMIS 2010 to characterize the epidemiology of wasting in children 0-23 months old in Bhutan, identify the most significant predictors of wasting, severe wasting and poor weight-forheight, and - on the basis of these findings - prioritize areas for action.

We found that almost one in ten children (9.3\%) aged 0-23 months was wasted and over one-third (37.6\%) of the wasted children were severely wasted. The prevalence of wasting was almost double among infants 0-11 months old than among children aged 12-23 months while the prevalence of severe wasting was $2 \cdot 4$-fold higher among infants 0-11 months old than among children aged 12-23 months, suggesting that most wasting happens either prenatally or in the first year of life. It has been documented that, compared with the WHO/National Center for Health Statistics 1976 reference, use of the WHO 2006 Child Growth Standards changes the age pattern of wasting in children of pre-school age, with significantly increased levels of wasting in early and late infancy $(0-5$ months and 6-11 months, respectively) than in early childhood (12-23 months) ${ }^{(13)}$.

Some of the most relevant findings of our analysis are related to the significant association of infant feeding 


\begin{tabular}{|c|c|c|}
\hline \multirow[b]{2}{*}{ Independent variable } & \multicolumn{2}{|c|}{$\begin{array}{l}\text { Dependent variable: } \mathrm{WHZ} \\
(0-23 \text { months old })\end{array}$} \\
\hline & Coefficient & $95 \% \mathrm{Cl}$ \\
\hline \multicolumn{3}{|l|}{ Age } \\
\hline $\begin{array}{l}\text { Child's age was } 0-11 \text { months } v \text {. Child's age was } 12-23 \text { months } \\
\text { Weighed at birth }\end{array}$ & -0.023 & $-0.170,0.120$ \\
\hline Child was weighed at birth $v$. Child was not weighed at birth & $0.187^{*}$ & $0.310,0.340$ \\
\hline Age-appropriate complementary feeding $(0-23)$ & & \\
\hline $\begin{array}{l}\text { Child was not fed CF when appropriate for age } v \text {. Child was fed CF when appropriate for age } \\
\text { Mother's education }\end{array}$ & 0.155 & $-0.040,0.358$ \\
\hline $\begin{array}{l}\text { Child's mother had no formal education } v \text {. Child's mother completed primary or secondary education } \\
\text { Wealth index }\end{array}$ & $0 \cdot 239^{*}$ & $-0.084,0.393$ \\
\hline $\begin{array}{l}\text { Child belonged to bottom } 50 \% \text { poorer households } v \text {. Child belonged to top } 50 \% \text { wealthier households } \\
\text { Region }\end{array}$ & -0.047 & $-0.201,0.107$ \\
\hline Child lived in the Western region $v$. Child lived in the Central/Eastern region & $-0 \cdot 230^{\star *}$ & $-0.381,-0.079$ \\
\hline
\end{tabular}

CF, complementary foods.

${ }^{\star} P<0.05,{ }^{* *} P<0.01$.

practices with wasting, severe wasting and attained WHZ. Poor complementary feeding practices - not aligned with internationally agreed-upon guidance - were the variables more systematically associated with wasting and severe wasting in children aged 0-23 months. Global and national policy recommends that infants aged $0-5$ months be exclusively breast-fed, with no other fluids or foods given, not even water, while children aged 6-23 months should be fed age-appropriate soft, semi-solid or solid complementary foods while breast-feeding continues ${ }^{(14)}$. In our sample, $28 \%$ of infants aged 6-8 months were not being fed complementary foods (timely introduction of complementary feeding), $23.9 \%$ of children aged 6-23 months were not being fed both breast milk and complementary foods (complementary feeding with continued breast-feeding) and $29.4 \%$ of children aged 6-23 months were not being fed complementary foods a minimum number of times per day (minimum meal frequency). Children 0-23 months old who were not fed complementary foods as recommended for their age had 1.6-fold higher odds of being wasted and 2.3-fold higher odds of being severely wasted after controlling for all other variables. This association was even stronger among infants aged 0-11 months: those who were not fed complementary foods as recommended for their age had twofold higher odds of being wasted and threefold higher odds of being severely wasted. A recent multi-country analysis of DHS data in eight countries found that several indicators of appropriate complementary feeding were positively associated with higher mean WHZ and/or lower odds of wasting in Uganda, Zambia and Zimbabwe ${ }^{(15)}$.

Prelacteal feeding (i.e. non-exclusive breast-feeding in the first $3 \mathrm{~d}$ of life) was associated with $2 \cdot 5$-fold higher odds of severe wasting in children 0-23 months old and 4.3-fold higher odds of severe wasting in infants 0-11 months old. However, age-appropriate breast-feeding (exclusive breastfeeding in infants aged $0-5$ months and continued breastfeeding in children aged 6-11 months) was associated with 2.5-fold higher odds of severe wasting in infants aged $0-11$ months $(\mathrm{AOR}=2 \cdot 51 ; 95 \% \mathrm{CI} 1 \cdot 13,5 \cdot 51)$. This finding is counter-intuitive and deserves further investigation. Studies in Bangladesh and Zambia found a positive association between exclusive breast-feeding and reduced odds of wasting ${ }^{(16,17)}$. Conversely, studies in Ethiopia, Haiti, India, Kenya, Uganda and Zimbabwe did not find any significant positive association between breast-feeding and lower odds of wasting or higher attained $\mathrm{WHZ}^{(15,17-19)}$. A recent metaanalysis on the growth benefits of breast-feeding has shown that breast-feeding interventions were associated with small, non-significant increases in weight and length/height $Z$-scores, and led to a modest, albeit significant, reduction in BMI $Z$-score/WHZ. For all outcomes, there was substantial heterogeneity among studies, which led the authors of the meta-analysis to indicate that their results 'must be interpreted with caution, (20).

However, the benefits of breast-feeding for child survival and development are well established ${ }^{(21,22)}$. Recent meta-analyses and systematic literature reviews by WHO show that children who are breast-fed have better survival rates, higher intelligence quotients, and lower risk of otitis, malocclusion, asthma and obesity. Breast-feeding mothers benefit from having breast-fed, with lower rates of breast cancer, ovarian cancer, type 2 diabetes and postpartum depression. The multiple benefits of breast-feeding demonstrate the contribution and relevance of breast-feeding as a global public health issue, for low- and high-income populations alike ${ }^{(23)}$. Therefore the protection, promotion and support of optimal breast-feeding needs to remain a central component of national programmes for child survival and development even if the empirical evidence of its direct impact on the prevention of wasting is limited ${ }^{(14,24)}$.

On the basis of our key findings we identify three policy and programme priorities:

1. Programmes for the detection and treatment of severely wasted children need to prioritize very young 
children (0-11 months), particularly in the Western region. Bhutan should consider adopting the community management of acute malnutrition (CMAM) approach for the early detection and care of children with severe wasting. Over fifty countries have adopted this approach ${ }^{(25,26)}$. CMAM should be scaled up as part of a continuum of care for the prevention and treatment of undernutrition in infancy and early childhood.

2. Programmes for the prevention of child wasting need to prioritize the improvement of complementary foods and feeding practices. There is agreement on the population-based indicators - timely introduction, feeding frequency, diet diversity, safe and responsive feeding - that should inform programme design and monitoring ${ }^{(27,28)}$. A nutrition surveillance system that provides information on these indicators - many of which were not included in BMIS 2010 - as well as information on seasonal and geographical variations in household food insecurity and child growth should inform a nationwide effort to improve children's access to age-appropriate nutritious complementary foods.

3. Programmes for the prevention of wasting need to ensure that women have access to information and support. While national efforts on girls' education are scaled up, attention needs to be provided to mothers with no/less formal education through supportive interventions like antenatal care. Our analysis shows that mothers' access to antenatal care was associated with a significantly lower risk of severe wasting in children, while being weighed at birth (a proxy for access to skilled delivery) was positively associated with WHZ in children. Antenatal and perinatal care provide an important platform to ensure that pregnant women receive counselling and support to improve the diets of their children in the first months of life.

Previous analyses have shown that better antenatal and perinatal care for women during pregnancy and delivery and appropriate complementary feeding for children in the first 2 years of life were also protective against child stunting in Bhutan ${ }^{(29)}$.

\section{Conclusion}

We analysed a nationally representative sample of 2028 children aged 0-23 months to characterize the epidemiology of wasting in Bhutan. We found that the prevalence of wasting was significantly higher among infants aged 0-11 months and children from the Western region. Poor feeding practices were among the most significant predictors of wasting and severe wasting, particularly among infants 0-11 months old. Prelacteal feeding in the first days of life and late introduction of complementary foods more than doubled the odds of severe wasting. Programmes for the prevention of child wasting in Bhutan need to prioritize the improvement of complementary foods and feeding practices in children aged 6-23 months, while programmes for the detection and treatment of severely wasted children need to prioritize very young children (0-11 months), particularly in the Western region.

\section{Acknowledgements}

Financial support: This research received no specific grant from any funding agency in the public, commercial or non-for-profit sectors. Conflict of interest: No conflicts to declare. Authorship: V.M.A. designed the study and led data analysis, data interpretation and manuscript writing; N.B. led data management; L.D. contributed to data interpretation. All authors have read and approved the final manuscript. Ethics of human subject participation: Ethical approval was not required. Disclaimer: The opinions expressed on this paper are those of the authors and do not necessarily represent an official position of the organizations with which they are affiliated.

\section{References}

1. Black RE, Victora CG, Walker SP et al.; Maternal and Child Nutrition Study Group (2013) Maternal and child undernutrition and overweight in low-income and middle-income countries. Lancet 382, 427-451.

2. WHO Multicenter Growth Reference Study Group (2006) WHO child growth standards based on length/height, weight and age. Acta Paediatr Suppl 450, 76-85.

3. Black RE, Allen LH, Bhutta ZH et al.; Maternal and Child Undernutrition Study Group (2008) Maternal and child undernutrition: global and regional exposures and health consequences. Lancet 371, 243-260.

4. Khara T \& Dolan C (2014) Associations between Wasting and Stunting. Policy, Programming and Research Implications. Technical Briefing Paper. London: Emergency Nutrition Network; available at http://files.ennonline.net/ attachments/1862/WAST_140714.pdf

5. Richard SA, Black RE, Gilman RH et al.; Childhood Infection and Malnutrition Network (2012) Wasting is associated with stunting in early childhood. J Nutr 142, 1291-1296.

6. Victora CG, Adair L, Fall C et al.; Maternal and Child Undernutrition Study Group (2008) Maternal and child undernutrition: consequences for adult health and human capital. Lancet 371, 340-357.

7. Dewey KG \& Vitta BS (2013) Strategies for Ensuring Adequate Nutrient Intake for Infants and Young Children during the Period of Complementary Feeding. AGT Technical Brief no. 7. Washington, DC: Alive and Thrive; available http://fr.cmamforum.org/Pool/Resources/ Insight-Issue-7-Ensuring-Adequate-Nutrition-2013.pdf

8. Chatterjee P (2007) Child malnutrition rises in India despite economic boom. Lancet 369, 1417-1418.

9. UNICEF (2014) The State of the World's Children 2015. New York: UNICEF; available at http://www.unicef.org/ publications/files/SOWC_2015_Summary_and_Tables.pdf

10. Bhutan National Statistics Bureau, UNICEF \& United Nations Population Fund (2011) Bhutan Multiple Indicator Survey 2010. Thimphu, Bhutan: National Statistics Bureau; available at http://www.ncwc.gov.bt/en/files/publication/ Bhutan\%20Multiple\%20Indicator\%20Survey\%20,\%202010.pdf 
11. Dewey KG \& Begum K (2011) Long-term consequences of stunting in early life. Maternal Child Nutr 7, 5-18.

12. Zangmo U, de Onis M \& Dorji T (2012) The nutritional status of children in Bhutan. Results from the 2008 National nutrition survey and trends over time. BMC Pediatrics 12, 151.

13. de Onis M, Onyango AW, Borghi E et al.; the WHO Multicentre Growth Reference Study Group (2006) Comparison of the World Health Organization (WHO) Child Growth Standards and the National Center for Health Statistics/ WHO international growth reference: implications for child health programmes. Public Health Nutr 9, 942-947.

14. World Health Organization (2012) Essential Nutrition Actions. Improving Maternal, Newborn, Infant and Child Health and Nutrition. Geneva: WHO; available at http:// apps.who.int/iris/bitstream/10665/84409/1/9789241505550_ eng.pdf

15. Jones AD, Ickes SB, Smith LE et al. (2014) World Health Organization infant and young child feeding indicators and their associations with child anthropometry. A synthesis of recent findings. Matern Child Nutr 10, 1-17.

16. Zongrone A, Winskell K \& Menon P (2012) Infant and young child feeding practices and child undernutrition in Bangladesh. Insights from nationally representative data. Public Health Nutr 15, 1697-1704.

17. Ali D, Rawat R, Subandoro A et al. (2012) Infant and young child feeding practices in Ethiopia and Zambia and their association with child nutrition. Analysis of demographic and health survey data. Afr J Food Agric Nutr Dev 12, 5895-5914.

18. Heidkamp R, Ayoya MA, Teta IN et al. (2015) Breastfeeding practices and child growth outcomes in Haiti. An analysis of data from Demographic and Health Surveys. Matern Child Nutr 11, 737-748.

19. Menon P, Bamezai A, Subandoro A et al. (2015) Age-appropriate infant and young child feeding practices are associated with child nutrition in India. Insights from nationally representative data. Matern Child Nutr 11, 73-87.

20. Giugliani ERJ, Horta BL, Loret de Mola C et al. (2015) Effect of breastfeeding promotion interventions on child growth.
A systematic review and meta-analysis. Acta Paediatr 104, 20-29.

21. Jones G, Steketee RW, Black RE et al. (2003) How many child deaths can we prevent this year? Lancet 362, 65-71.

22. Bhutta ZA, Das JK, Rizvi A et al.; Maternal and Child Nutrition Study Group (2013) Evidence-based interventions for improvement of maternal and child nutrition: what can be done and at what cost? Lancet 382, 452-477.

23. Grummer-Strawn LM \& Rollins N (2015) Summarising the health effects of breastfeeding. Acta Paediatr 104, 1-2.

24. Jimenez M \& Stone-Jimenez M (2014) Preventing moderate acute malnutrition (MAM) through nutrition-specific interventions. CMAM Forum Technical Brief. http://www. cmamforum.org/Pool/Resources/Nutrition-specific-MAMprevention-CMAM-Forum-Technical-Brief-Sept-2014-.pdf (accessed December 2015).

25. Emergency Nutrition Network (2012) Special Focus on Government Experiences of Community Management of Acute Malnutrition (CMAM) Scale Up. Field Exchange issue 43. London: ENN; available at http://www.cmamforum.org/ Pool/Resources/fx-43-govt-experiences-with-CMAM-scaleup-part-1-2012(3).pdf

26. World Health Organization \& UNICEF (2009) WHO Child Growth Standards and the Identification of Severe Acute Malnutrition in Infants and Children. A Joint Statement by WHO and UNICEF. Geneva: WHO; available at http://apps. who.int/iris/bitstream/10665/44129/1/9789241598163_eng. pdf?ua=1http://

27. Daelmans B, Dewey K \& Arimond M (2009) New and updated indicators for assessing infant and young child feeding. Food Nutr Bull 30, 2 Suppl., S256-S262.

28. World Health Organization (2008) Indicators for Assessing Infant and Young Child Feeding Practices (Part I): Definitions. Geneva: WHO; available at http://www.unicef. org/nutrition/files/IYCF_Indicators_part_III_country_profiles. pdf

29. Aguayo VM, Badgaiyan N \& Paintal K (2015) Determinants of child stunting in the Royal Kingdom of Bhutan. An indepth analysis of nationally representative data. Matern Child Nutr 11, 333-345. 